\title{
Modeling Coffee Price using Jump Diffusion Model: The case of Ethiopia
}

\author{
Tesfahun Berhane, Molalign Adam, Guriju Awgichew and Eshetu Haile
}

\begin{abstract}
Ethiopian coffee price has significant effect on the economy of the country and its price is highly fluctuated. In this study, we aim at modeling and forecasting the washed Sidama class A grade 3 (WSDA3) coffee price in Ethiopia to reduce the risks associated with this price fluctuation. We used daily closed price data of Ethiopian WSDA3 coffee recorded in the period 31 May 2011 to 30 March 2018 obtained from Ethiopia commodity exchange (ECX) market to analyse the prices fluctuation. The nature of log-returns of the price is asymmetric (negatively skewed) and exhibits high kurtosis. We used a Jump diffusion model to model and forecast the empirical data. The method of maximum likelihood is used to estimate the parameters. We used the root mean square error (RMSE) to test the goodness of fitting for the model to the data. This test indicates that the model performs well.
\end{abstract}

Index Terms-Jump diffusion, maximum likelihood, logreturn, Ethiopian coffee.

\section{INTRODUCTION}

$\mathbf{C}$ URRENTLY, agricultural sector has great contribution to the development of the Ethiopian economy. Specially, agricultural products that have been sold at world market level (export products) are of great importance. Coffee is one of such agricultural products which play a vital role in Ethiopian economy. Ethiopia is the origin for coffee Arabica. The country possesses a diverse genetic base for this Arabica coffee with considerable heterogeneity. Ethiopia produces a range of distinctive Arabica coffees and has considerable potential to sell a large number of specialty coffees [1]. Coffee production in Ethiopia is almost exclusively situated in two regions of the country: Oromia and the Southern Nations, Nationalities, and People Regions (SNNPR) in the south and west of the country (see Fig. 1).

Coffee dominates export revenues in Ethiopia; such a pattern will not change in the short period of time. It is the major agricultural export crop, providing $65 \%$ of Ethiopia's foreign exchange earnings [2]. Coffee is the most important cash crop in Ethiopia, with more than 15 million people directly or indirectly depending on it for their livelihoods [3]. For this reason, the Ethiopian government has taken different amendment to improve the productivity, quality and market efficacy of its domestic coffee crops. As a result, Ethiopia becomes the largest coffee producer and exporter in Africa and the world's tenth major coffee exporters. The country

Manuscript received July 16, 2018; accepted January 13, 2019.

The authors are with the Department of Mathematics, College of Science, Bahir Dar University, P.O.B 79, Ethiopia. E-mails: tesfahunb2002@gmail.com, molalign2@yahoo.com, fevenjerry@gmail.com, eshetuhg@gmail.com

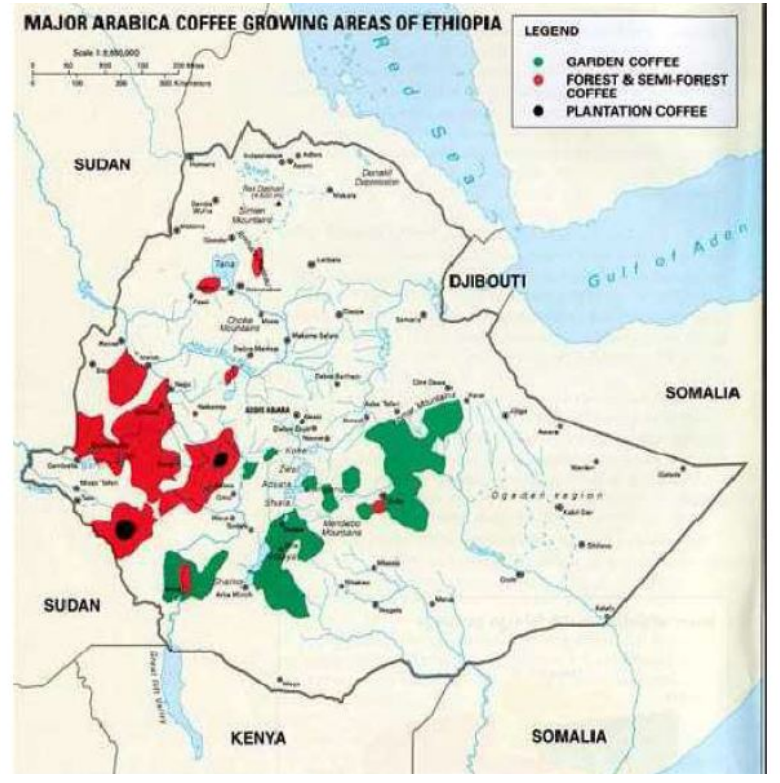

Fig. 1. Major Ethiopian coffee producing areas.

exported 3.2 million bags of coffee and accounted for $3 \%$ of internationally traded coffee [4].

Global coffee production varies from year to year according to weather conditions, diseases and other factors, resulting in a coffee market that is inherently unstable and characterized by wide fluctuations in price. This price volatility has significant consequences for those who depend on coffee for their livelihood, making it difficult for farmers to predict their income for the coming season and budget for their household and farming needs. Ethiopian coffee prices are often varies as they are largely influenced by production, demand of coffee in domestic and world level forces, quality of products, etc. Therefore, it is of great significance to develop a model to reduce the risks associated with Washed Sidama class A Grade 3 (WSDA3) coffee prices fluctuations.

In spite of the success of the Black-Scholes model based on Brownian motion and normal distribution, the empirical phenomena has received much attention recently on the asymmetric leptokurtic features. In other words, the return distribution is skewed to the left, and has a higher peak and heavier tails than those of the normal distribution. Thus, many models have been proposed in order to reflect the above phenomena. Most of the research has focused on the leptokurtic feature under the market measure. Merton jump diffusion model is one of a special case of affine jump diffusion model which is used to capture the leptokurtic feature of an asset price [5]. 
The log-returns of WSDA3 coffee prices are characterized by large fluctuations in value. Besides, the empirical distribution of log-returns is asymmetric (negatively skewed) and exhibits high kurtosis.

According to the knowledge of the authors, no study has been done for Ethiopian coffee prices for modeling and forecasting using stochastic differential equations. Therefore, we apply Jump diffusion model to solve the problem by using the discontinuities by a Poisson process with BlackScholes model. We use the method of maximum likelihood to estimate the parameters for Merton-Jump Diffusion (MJD) model. Based on the empirical results that we obtained, we show the model fitting and forecasting to the empirical data. Finally, we use the root mean square error (RMSE) to test the model validation.

\section{ANALYSIS OF WSDA3 COFFEE PRICE DATA}

The daily closed price of Ethiopian coffee specifically WSDA3 from 31 May 2011 to 30 March 2018 recorded by Ethiopia commodity exchange (ECX) market is considered to study WSDA3 coffee price movements.

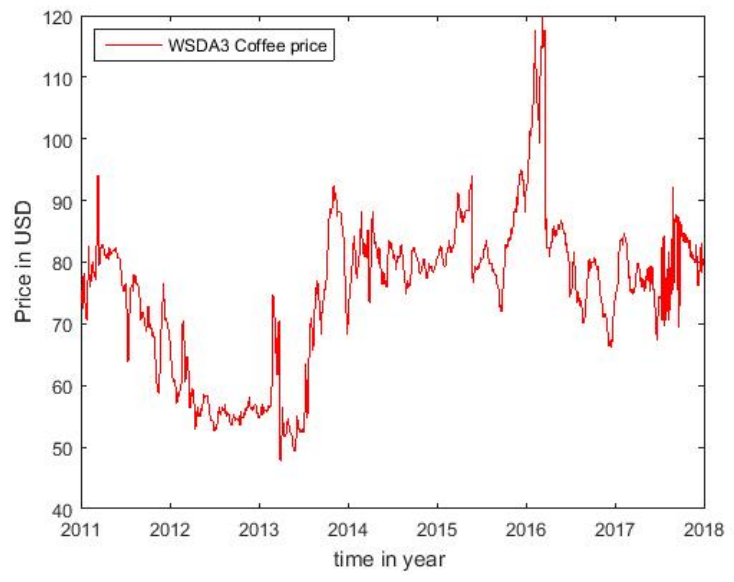

Fig. 2. The WSDA3 coffee price from 2011 to 2018.

\section{A. Autocorrelation Functions (ACF)}

In general, the WSDA3 coffee price plot shows that the price does not have any seasonal pattern. The autocorrelation function (ACF) is an important diagnostic tool for analyzing time series in the time domain [6]. The ACF is also very useful when examining stationarity and when selecting from various non-stationary models. In autocorrelation, lag is a time period separating the ordered data and is used to calculate the autocorrelation coefficients. Autocorrelation plots, called the correlograms, present a better understanding of the evolution of a process through time using the probability of the relationship between data values separated by a specific number of time steps (lags). The ACF correlogram of WSDA3 coffee price from 2011 to 2018 in Fig. 3 shows that the autocorrelation coefficients decrease to zero as the lag increases and this is a non-stationary time series. This result shows that WSDA3 coffee price data has no seasonal pattern.

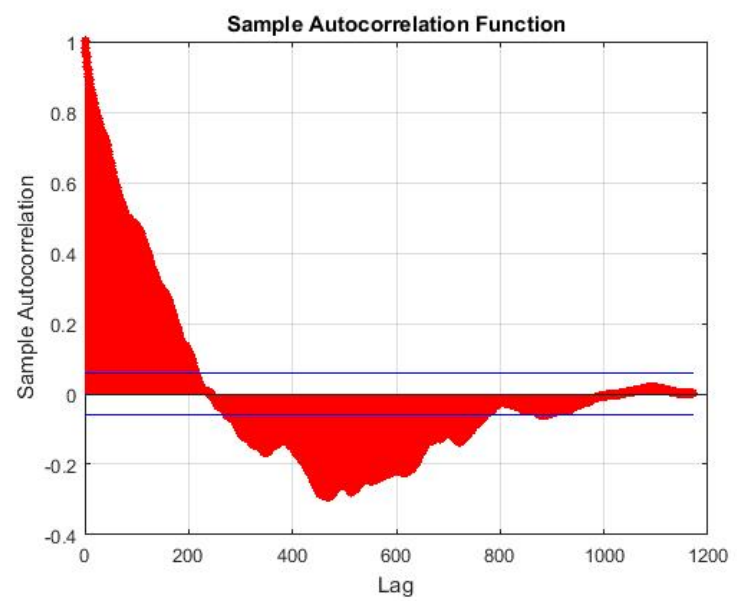

Fig. 3. The ACF for coffee price from 2011 to 2018.

\section{B. Normality test}

Consider $S_{t}$ be the daily recorded closing WSDA3 coffee prices from 31 May 2011 to 30 March 2018. We insight into the coffee price dynamics which is gained by analyzing logprice return defined as:

$$
x_{t}=\Delta \ln \left(S_{t}\right)=\ln \left(S_{t}\right)-\ln S_{t-}
$$

We plot the histogram together with the normal density in Fig. 4. This figure shows that the presence of fat tails and high kurtosis in the empirical distribution of WSDA3 coffee price. Thus, the price of Ethiopian WSDA3 coffee is not normally distributed.

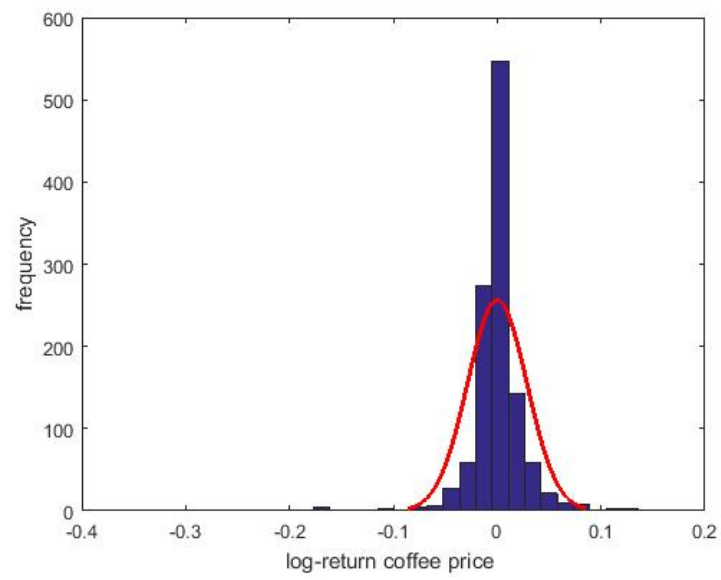

Fig. 4. The histogram and fitted normal density of daily log-return WSDA3 coffee price from 31 May 2011 to 30 March 2018.

In Fig. 5, the Q-Q plot of the daily log-return WSDA3 cofee price from 31 May 2011 to 30 March 2018 is not linear. Therefore, the distribution of the daily return is not normally distributed.

\section{Comparison of Global coffee prices and Ethiopian WSDA3 Coffee prices}

The Global coffee price is characterized by intercontinental commodity exchanges. Also, like any other exchange-traded 


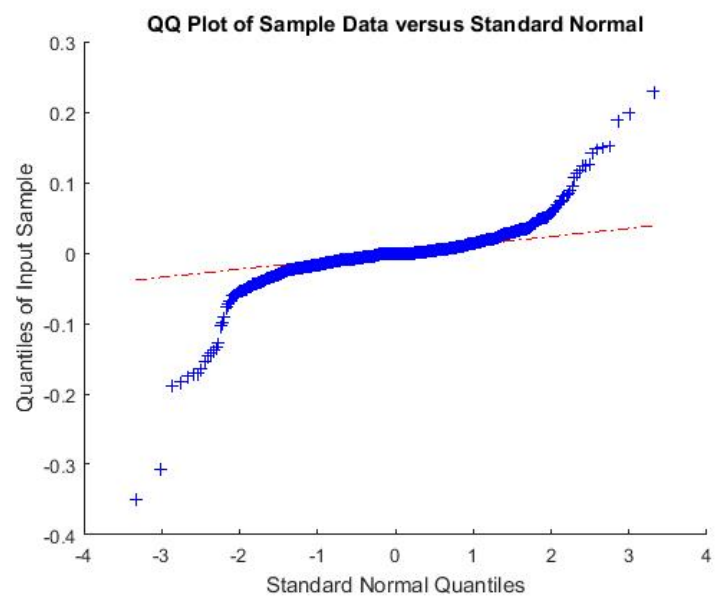

Fig. 5. Q-Q plot of log-return 3 coffee price from 31 May 2011 - 30 March 2018.

TABLE I

WDSA3 COFFEE LOG-PRICE DESCRIPTIVE STATISTICS

\begin{tabular}{|c|c|}
\hline Descriptive statistics & Value \\
\hline Mean & 4.302975837785977 \\
\hline Standard Deviation & 0.170556112066934 \\
\hline Skewness & -0.522427157268832 \\
\hline Kurtosis & 2.878948109483763 \\
\hline
\end{tabular}

commodity, stock, bond, currency, etc. It is determined by supply and demand. The dynamic behavior of price of the Global coffee and WSDA3 coffee starts to increase from 2011 till the mid of 2011. Again, the Global coffee price shows a rapidly decreasing from the mid of 2011 till the end of 2013. At the same time, the WSDA3 coffee price exhibits the same behavior in this period. However, both the Global and WSDA3 coffee prices drastically increase in the period of 2014. Then the price of Global coffee gradually decreases up to the end of 2015 and it starts rise up till the mid of 2016. Finally, it decreases gradually to the end of 2017. Also the WSDA3 coffee price falls down to the end of 2015 and then it goes up to the mid of 2016. Lastly, it gradually decreases till the end of 2017. In general, in this discussion we conclude that the dynamic behavior of Global coffee has a great impact on the prices of Ethiopian WSDA3 coffee.

\section{MODEL}

Based on the empirical findings discussed in the previous section, namely the presence of skewness and kurtosis in the empirical distribution of WSDA3 coffee prices, returns, an adequate model for WSDA3 coffee prices would be a jump diffusion model.

In fact, Merton [7], recognizing the presence of jumps in asset prices and for more accurate option pricing, proposed modeling these prices as a jump diffusion process instead of a pure diffusion model. Pure diffusion based models could not adequately explain the smile effect in short-dated option prices and emphasized the importance of adding a jump component in modeling asset price dynamics [8].
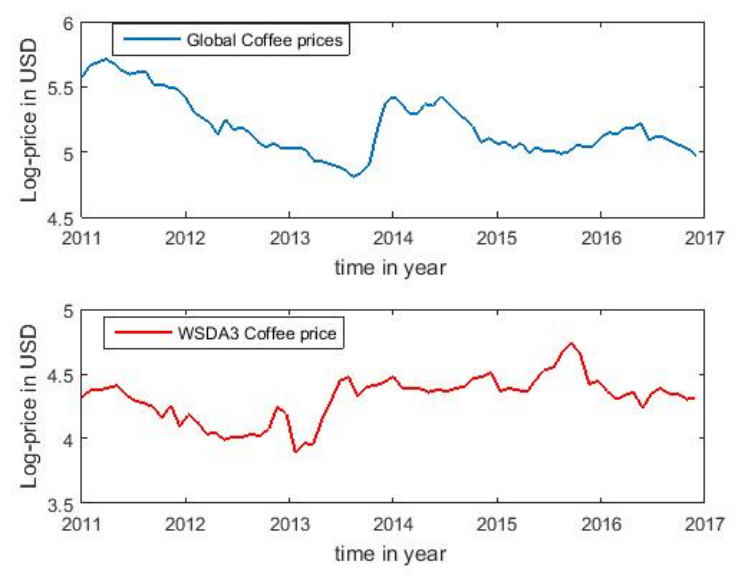

Fig. 6. Monthly log-prices of Global and WSDA3 coffee prices.

Models with jumps generically lead to significant skews for short-term maturities. More generally, adding jumps to returns in a diffusion-based stochastic volatility model, the resulting model can generate sufficient variability and asymmetry in the short-term returns to match implied volatility skews for shortterm maturities [9]. Hence, the price process $S_{t}$ is assumed to follow the stochastic differential equation.

$$
\frac{d S_{t}}{S_{t-}}=\mu d t+\sigma d B_{t}+\left(y_{t}-1\right) d N_{t}
$$

where $S_{t}$ denotes the coffee price, $\mu$ is instantaneous expected return and $\sigma$ is the instantaneous volatility of the price return. The continuous component is given by a standard Brownian motion, $B_{t}$, distributed as $d B_{t} \sim(0, d t)$. The discontinuities of the price process are described by a Poisson counter $N_{t}$, characterized by its intensity, $\lambda$, and jump size $y_{t}$. The assumption is that the Brownian motion $B_{t}$, the Poisson process $N_{t}$ and the jump size $y_{t}$ are independent. The probability that the price jumps during a small time interval $d t$ can be written using a Poisson process $\Delta N_{t}$ as:

$$
\begin{aligned}
& \operatorname{Prob}\left[\Delta N_{t}=1\right]=\lambda d t, \quad \text { and } \\
& \operatorname{Prob}\left[\Delta N_{t}=0\right]=1-\lambda d t,
\end{aligned}
$$

where $\lambda$ is the intensity of the jump process (the mean number of jumps per unit time). When abnormal information arrives, WSDA3 coffee prices jumps from $S_{t-}$ (limit from left) to $\left.S_{t}=y_{t} S_{(} t-\right)$. The percentage change is measured by $\left(y_{t}-1\right)$. The price $S_{t}$ presents log-normal jumps $y_{t}$ on each random time $t$ which represents the moments of jumping of a Poisson process [10], [11], [12]. Introduction of the Jump diffusion model adds three extra parameters $\left(\beta, \delta^{2}, \lambda\right)$ to the BlackScholes process model which contains two parameters $\left(\mu, \sigma^{2}\right)$. Merton assumes that the log-price jump size $Y_{t}=\ln \left(y_{t}\right)$ is normal random variables. Letting $X_{t}=\ln \left(S_{t}\right)$ and using Ito's lemma, the log-price return process becomes:

$$
d X_{t}=\left(\mu-\frac{1}{2} \sigma^{2}\right) d t+\sigma d B_{t}+Y_{t} d N_{t}
$$


Discretized over $[t, t+\Delta t]$, this model takes the form:

$$
\Delta X_{t}=\left(\mu-\frac{1}{2} \sigma^{2}\right) \Delta t+\sigma \Delta B_{t}+\sum_{j=0}^{\Delta N_{t}} Y_{j}
$$

where $\Delta B_{t}=B_{t+\Delta t}-B_{t} \sim N(0, \Delta t)$ and $\Delta N_{t}=N_{t+\Delta t}-N_{t}$ is the number of jumps occurring during the time interval over $(t, t+\Delta t)$ and $Y_{t}$ are independently and identically distributed as $Y_{t} \sim N\left(\beta, \delta^{2}\right)$. The log-return, $x_{t}=\Delta X_{t}$, therefore includes the sum of two independent components: a diffusion component with drift and a jump component. The probability density of $\Delta X_{t}$ can be expressed [9] as

$$
\begin{array}{r}
f(x)=\sum_{n=0}^{\infty} \frac{(\lambda \Delta t)^{n} e^{-\lambda \Delta t}}{n !}\left[\frac{1}{\sqrt{\left(2 \pi\left(\sigma^{2} \Delta t+n \delta^{2}\right)\right)}}\right. \\
\left.\exp \left(-\frac{\left(x-\left(\mu-\frac{1}{2} \sigma^{2}\right) \Delta t-n \beta\right)^{2}}{2\left(\sigma^{2} \Delta t+n \delta^{2}\right)}\right)\right]
\end{array}
$$

with $n=0,1,2, \ldots$. Putting $\Delta t=1$ that is the time interval is $(t, t+1)$, the density function becomes

$$
\begin{array}{r}
f(x)=\sum_{n=0}^{\infty} \frac{(\lambda)^{n} e^{-\lambda}}{n !}\left[\frac{1}{\sqrt{\left(2 \pi\left(\sigma^{2}+n \delta^{2}\right)\right)}}\right. \\
\left.\exp \left(-\frac{\left(x-\left(\mu-\frac{1}{2} \sigma^{2}\right)-n \beta\right)^{2}}{2\left(\sigma^{2}+n \delta^{2}\right)}\right)\right]
\end{array}
$$

\section{PARAMEter Estimation}

Let $x=\left\{x_{1}, x_{2}, x_{3}, \ldots, x_{T}\right\}$ be an observed sample of logreturns, the log-likelihood function can be expressed as:

$$
\begin{array}{r}
L(x ; \theta)=-T \lambda-\frac{T}{2} \ln (2 \pi)+\sum_{n=0}^{T} \ln \left[\sum _ { n = 0 } ^ { \infty } \frac { \lambda ^ { n } } { n ! } \left(\frac{1}{\sqrt{\left(\sigma^{2}+n \delta^{2}\right)}}\right.\right. \\
\left.\left.\exp \left(-\frac{\left(x-\left(\mu-1 / 2 \sigma^{2}\right)-n \beta\right)^{2}}{2\left(\sigma^{2}+n \delta^{2}\right)}\right)\right)\right]
\end{array}
$$

where the parameter vector associated with the price process is therefore $\theta=(\mu, \sigma, \beta, \delta, \lambda)$. These parameters are estimated for the model using the method of maximum likelihood estimation (MLE) on log-likelihood function specified in (6).

We consider the first 999 log-return WSDA3 coffee price for parameters estimation. We truncate the number of jumps at $n=10$ to estimate the parameter values as it is pointed out by [13].

In this paper, we used maximum likelihood estimation technique to estimate the parameters with corresponding $95 \%$ confidence intervals for each parameter and the values are presented in Table 2. In Table 2, Lb denotes the lower bound and $\mathrm{Ub}$ the upper bound of confidence intervals.

\section{Model Simulation}

In this section, we used the Euler discretized version of the model specified in (2) to simulate the WSDA3 coffee price.
TABLE II

ESTIMATED PARAMETERS FOR THE MODEL

\begin{tabular}{|c|c|c|c|}
\hline \multirow{2}{*}{ Parameters } & \multirow{2}{*}{$\begin{array}{l}\text { 95 \% Confidence } \\
\text { Interval }\end{array}$} \\
\cline { 3 - 4 } & Values & Lb & Ub \\
\hline$\mu$ & 0.0003114 & -0.0005290 & 0.00115 \\
\hline$\sigma$ & 0.0087656 & 0.0071501 & 0.0103810 \\
\hline$\beta$ & -0.0004219 & -0.0042323 & 0.0033884 \\
\hline$\delta$ & 0.0344160 & 0.0270214 & 0.0418105 \\
\hline$\lambda$ & 0.3864923 & 0.2342795 & 0.5387050 \\
\hline
\end{tabular}

The discretized form of the model over the time interval $(t, t+$ 1) can be expressed as:

$$
X_{t+1}=X_{t}+\left(\mu-\frac{1}{2} \sigma^{2}\right) t+\sigma B_{t}+\sum_{j=0}^{N_{t}} Y_{j}
$$

Replacing $\ln \left(S_{t}\right)$ for $X_{t}$ and $\ln \left(S_{t+1}\right)$ for $X_{t+1}$, we obtain:

$$
\ln \left(S_{t+1}\right)=\ln \left(S_{t}\right)+\left(\mu-\frac{1}{2} \sigma^{2}\right) t+\sigma B_{t}+\sum_{j=0}^{N_{t}} Y_{j}
$$

where $B_{t} \sim N(0,1)$. This implies that

$$
\left.S_{t+1}=S_{t} \exp \left(\mu-\frac{1}{2} \sigma^{2}\right) t+\sigma B_{t}+\sum_{j=0}^{N_{t}} Y_{j}\right)
$$

We used the model specified in (8) and in (9) for the simulation of Ethiopian coffee prices. The fitted values from the simulation are plotted against the observed coffee price in Fig. 7. Also, we plot both the observed and forecasted coffee price in Fig. 8.

\section{RESUlts AND Discussions}

In this study, we use daily recorded WSDA3 coffee price from 31 May 2011 to 30 March 2018 from the Ethiopian Commodity Exchange (ECX). The method of maximum likelihood is being used to estimate the parameters as shown in Table 2. This result indicated that the dynamics of the price process were influenced by both diffusion and jump components. However, the price was dominated by a jump component with large discontinuities occurring at high intensity. The high volatility of the jump component reflects the presence of jumps of large magnitude and is in accordance with excess kurtosis in the empirical distribution of the data.

The mean of the jump size tended to be negative, in conformity with negative skewness in the empirical distribution. Also, the intensity of the jump process which is estimated up on using the method is high and significant, indicating that the price process was characterized by frequent jumps.

We used the model to forecast the WSDA3 coffee prices for 174 days ahead. Figure 8 shows that the model is efficient to forecast this price. We used the root mean square error (RMSE) to test the validity of the model and we obtain RMSE 0.163155215377826. This result indicates that the model performs well. 

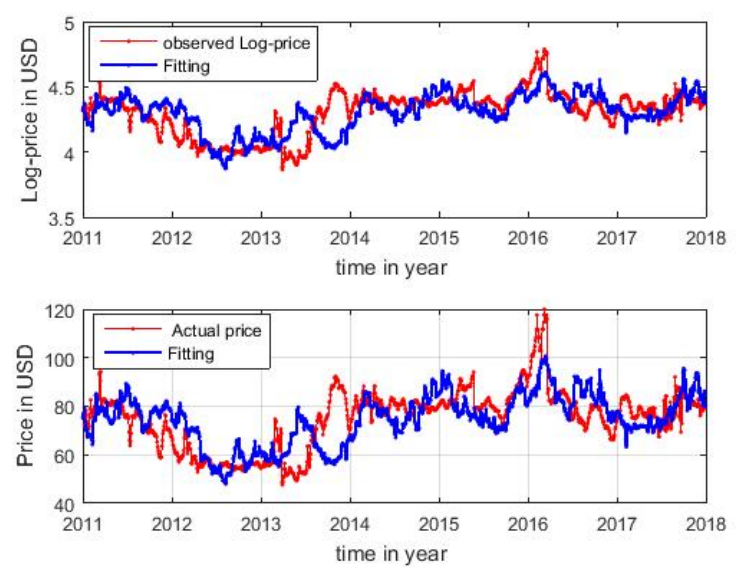

Fig. 7. Fitting with log-price of WSDA 3 coffee (Top panel) and fitting with observed WSDA3 price of coffee (Bottom panel) from 2011 to 2018.

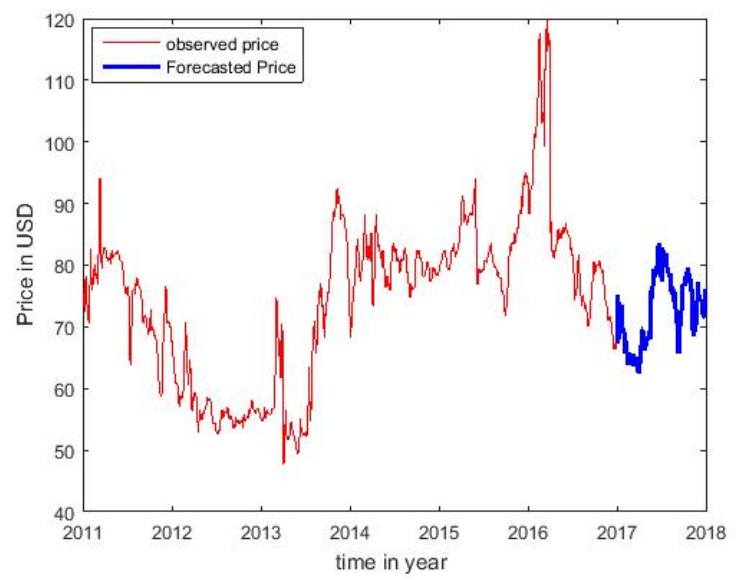

Fig. 8. Observed WSDA3 coffee price and forecasted price.

\section{CONCLUSIONS}

WSDA3 coffee prices from 2011 to 2018 are characterized by large fluctuations in value. From Fig. 6, we deduce that the dynamic behavior of global coffee price has an impact on Ethiopian WSDA3 coffee price fluctuations. The nature of log-returns of the coffee price is asymmetric (negatively skewed) and exhibits high kurtosis. We apply a suitable model for modeling and forecasting to the empirical data to capture the dynamic behavior of WSDA3 coffee price. The method of maximum likelihood is used to estimate the parameters. We used the root mean square error (RMSE) to test the goodness of fitting for the model to the empirical data. The result indicates that the model performs well. This model will be applied for further problems based on the stochastic differential equations with jumps to derive the analytical expression of the future prices for some asset prices.

\section{REFERENCES}

[1] D. Nure, "Mapping quality profiles of ethiopian coffee by origin," $E d s$. Adugna, G., Bellachew, B., Shimber, T., Taye, E., Kufa, pp. 317-227, 2008.
[2] B. Beshah, D. Kitaw, and T. Dejene, "Quality and value chain analyses of ethiopian coffee," Journal of Agriculture and Social Research (JASR), vol. 13, no. 2, pp. 35-41, 2013.

[3] G. A. I. N. (GAIN), "Coffee annual report:assessment commodity and trade issue made by usda staff," GAIN Report no. ET-1615, Tech. Rep., 2016.

[4] B. Minten, S. Tamru, T. Kuma, and Y. Nyarko, Structure and performance of Ethiopia s coffee export sector. Intl Food Policy Res Inst, 2014, vol. 66

[5] F. Hanson and J. Westman, "Stochastic analysis of jump-diffusions for financial log-return processes," in Stochastic Theory and Control, 2002, pp. $169-183$.

[6] J. Rafiee and P. Tse, "Use of autocorrelation of wavelet coefficients for fault diagnosis," Mechanical Systems and Signal Processing, vol. 23, no. 5, pp. 1554-1572, 2009.

[7] R. Merton, "Option pricing when underlying stock returns are discontinuous," Journal of financial economics, vol. 3, no. 1-2, pp. 125-144, 1976.

[8] G. Sarwar and T. Krehbiel, "Empirical performance of alternative pricing models of currency options," Journal of Futures Markets: Futures, Options, and Other Derivative Products, vol. 20, no. 3, pp. 265-291, 2000.

[9] H. Askari and N. Krichene, "Oil price dynamics (2002-2006)," Energy Economics, vol. 30, no. 5, pp. 2134-2153, 2008.

[10] E. Jondeau, S.-H. Poon, and M. Rockinger, Financial modeling under non-Gaussian distributions. Springer Science \& Business Media, 2007.

[11] P. Jorion, "On jump processes in the foreign exchange and stock markets," The Review of Financial Studies, vol. 1, no. 4, pp. 427-445, 1988.

[12] S. Kou, "A jump-diffusion model for option pricing," Management science, vol. 48, no. 8, pp. 1086-1101, 2002.

[13] C. Ball and W. Torous, "On jumps in common stock prices and their impact on call option pricing," The Journal of Finance, vol. 40, no. 1, pp. 155-173, 1985. 